\title{
Freiheit und Sicherheit nach dem 11. September 2001 - Versuch einer Zwischenbilanz
}

\section{Die Taten und ihre Besonderheiten}

Das, was am 11. September 2001 in den Vereinigten Staaten von Amerika geschah, ist mit gutem Grund als »Weltereignis« bezeichnet worden. Die monströsen Taten wurden nicht nur von langer Hand an verschiedenen Orten der Welt vorbereitet, sie fanden zudem Nachahmung an unterschiedlichsten Orten der Erde. Auf die Flugzeugabstürze von New York, Washington und Pennsylvania folgten die Attentate von Djerba (April 2002), Santos (April 2002), Karachi (Juni 2002), Bali (Oktober 2002), Mombasa (November 2002), Riad (Mai und November 2003), Casablanca (Mai 2003), Jakarta (August 2003), Istanbul (November 2003), Madrid (März 2004), London (Juli 2005) und Amman (November 2005). Diese Attentate, deren Aufzählung keinesfalls vollständig ist und die inzwischen mehreren Tausend Zivilisten das Leben kosteten, wurden regelmäßig von religiös motivierten politischen Gewalttätern begangen, die den eigenen Tod als festen Bestandteil des Tatgeschehens einplanten. Diese Todesbereitschaft unterscheidet sie von den terroristischen Gewalttätern der siebziger und achtziger Jahre des vergangenen Jahrhunderts. Gewiss konnten auch die Mitglieder der italienischen Brigate Rosse, der belgischen Communistes Cellules Combattantes oder der deutschen Roten-Armee-Fraktion bei ihren Taten den eigenen Tod nicht ausschließen. Sie suchten ihn aber zu vermeiden, wenn auch nur, um für den weiteren Kampf zur Verfügung zu stehen. Insoweit war ihr Handeln zumindest bis zu einem gewissen Grad kalkulierbar. Aber selbst ein solches Maß an Berechenbarkeit löst sich auf, wenn Täter ihren Selbsterhaltungstrieb mit Hilfe religiös-spiritueller Jenseitserwartungen überwinden und im Attentat auch den eigenen Tod wollen.

Als Spezifikum der terroristischen Täter der Gegenwart sticht außerdem hervor, dass es ihnen gelang, sich jahrelang im Schutz westlicher rechtsstaatlicher Legalität zu bewegen, ohne den Sicherheitsbehörden Anlässe zu Überprüfungs- und Kontrollmaßnahmen zu liefern. Das tatvorbereitende Handeln dieser so genannten »Schläfer« begründete weder eine polizeiliche Gefahr, noch einen strafprozessualen Anfangsverdacht. Ebensowenig waren die Voraussetzungen von Vorfeldbefugnissen gegeben, setzen diese doch, wie etwa Observationen oder sonstige Datenerhebungen zum Zwecke der vorbeugenden Verbrechensbekämpfung, regelmäßig früheres auffälliges Verhalten voraus. Ein solches Verhalten legte etwa die mehrere Jahre lang in Hamburg lebende Terroristenzelle, die an den Attentaten in den USA beteiligt war, aber gerade nicht an den Tag.

* Bei dem folgenden Text handelt es sich um eine aktualisierte und geringfügig ergänzte Fassung eines Beitrags, der für das Rundschreiben des Bundes der deutschen Verwaltungsrichter (BDVR 2/2004) verfasst wurde. Dieses Rundschreiben erschien anlässlich des 14. Verwaltungsrichtertages 2004 in Bremen. 


\section{Fragen und Antworten}

Die mit und seit dem 11. September 2001 sichtbar gewordene neue Gefährdungslage hat auch die Bundesrepublik Deutschland mit einer Fülle sicherheitsrechtlich bedeutsamer Fragen konfrontiert, nicht zuletzt, weil wesentliche Vorbereitungshandlungen in Deutschland stattfanden und unter den Opfern auch zahlreiche deutsche Staatsangehörige waren. Diese Fragen betreffen den Bestand an Handlungsermächtigungen der Sicherheitsbehörden, deren Organisationsstruktur, die Leitkonzepte des Sicherheitsrechts sowie, vor allem, das Verhältnis von Sicherheit und Freiheit.

Zunächst: Verfügen die Sicherheitsbehörden über ausreichende Handlungsinstrumente? Sind neue Straftatbestände zu schaffen, neue Verbotsnormen und neue Ermittlungsbefugnisse? Sodann: Ist die Organisation der Sicherheitsbehörden der neuen Gefahrenlage noch angemessen? Ist etwa eine neue Sicherheitsarchitektur das Gebot der Stunde? Sollte der Polizei- und Verfassungsschutzföderalismus beschränkt, die Polizeien des Bundes gestärkt, deren kriminalpolizeiliche Komponenten in Gestalt des Bundeskriminalamtes, des Zollkriminalamtes sowie des inzwischen zur Bundespolizei umbenannten Bundesgrenzschutzes vielleicht zu einer eigenen Bundeskriminalpolizei gebündelt werden? Ist das aus der Besatzungszeit herrührende Gebot der Trennung von Polizei und Nachrichtendiensten aufzugeben, wenigstens partiell? Wie leistungsfähig und intelligent ist die in den Anfangszeiten des Kalten Krieges wurzelnde verfassungsrechtliche Differenzierung zwischen der auf den Verteidigungsfall bezogenen Zivilschutzkompetenz des Bundes und der friedenszeitlichen Katastrophenschutzzuständigkeit der Länder und Kommunen? Sind neue Ermächtigungen für den Einsatz der Streitkräfte im Innern zu schaffen, etwa zur Abwehr von Angriffen von See her, zur Dekontaminierung im Falle terroristischer Attacken mit ABC-Waffen oder zum Schutz von Polizeibeamten oder Sanitätskräften, die sich in ein freies Schussfeld begeben müssen? Ist die internationale Zusammenarbeit der Sicherheitsbehörden weiter zu intensivieren und auszubauen, sind neue überstaatliche Sicherheitsbehörden zu errichten und ein europäisch-transnationales, die Souveränitätsgrenzen überwindendes Tätigkeitsfeld nationalstaatlicher Sicherheitsorgane zu schaffen? Schließlich: Ist das traditionelle Sicherheitsrecht, das auf den systemtragenden Leitkategorien der Gefahr sowie des Straftatenverdachts fußt, zugunsten eines Sicherheitsvorsorge- und Präventionsrechts zu reformulieren, das noch weiter als bisher in die Vorstadien einer Gefahr oder eines Tatverdachts hinausgreift - gerade weil die abschreckende Wirkung des repressiven Strafrechts den zur Selbsttötung bereiten Attentäter verfehlt und es zudem viel zu riskant ist, solange zu warten, bis sich angesichts zu allem entschlossener Täter eine Gefährdungslage zu einer schadensnahen Gefahr verdichtet hat? Muss dementsprechend auch Sicherheit neu gedacht werden? Hat etwa der Begriff der inneren Sicherheit als Ordnungsbegriff ausgedient, weil angesichts der neuen Lage die ihm zugrunde liegende Unterscheidung von Innen und Außen an Überzeugungskraft verloren hat? Ist er durch einen erweiterten Sicherheitsbegriff zu ersetzen, der nicht an eine Gefahr anknüpft und sich auch nicht auf Gefahrenvorsorge beschränkt, der vielmehr schon plausible Erwartungen aufgrund von Gefahrenanalysen oder Gefährdungsschätzungen mit einbezieht, die auch auf wirtschaftlichen, finanziellen, sozialen und sonsti- 
gen Faktoren beruhen können? Und nicht zuletzt: In welchem Maße sind Einschränkungen der verfassungsrechtlich verbürgten Freiheitsrechte hinzunehmen, angesichts des sich zumindest auf den ersten Blick aufdrängenden Eindrucks, dass jedes Mehr an Sicherheit zu einem Weniger an Freiheit führt? Kurz: Muss das spannungsträchtige Verhältnis von Freiheit und Sicherheit neu justiert werden und, falls ja, wie?

Bund und Länder haben seit dem 11. September 2001 auf diese Fragen zu antworten versucht, zunächst durch eine Verbesserung der finanziellen und personellen Ausstattung der Sicherheitsorgane, dann aber vor allem mit legislativen Maßnahmen. Im Einzelnen sind die folgenden Gesetze zu nennen, die allesamt mit der Notwendigkeit gerechtfertigt wurden, auf die neue Gefährdungslage reagieren zu müssen:

- Erstes Gesetz zur Änderung des Vereinsgesetzes (BGBl. I 2001, S. 3319),

- Gesetz zur Finanzierung der Terrorbekämpfung (BGBl. I 2001, S. 3436),

- Gesetz zur Bekämpfung des internationalen Terrorismus (Terrorismusbekämpfungsgesetz) (BGBl. I 2002, S. 361 ff.), damit verbunden die Gesetze der Länder zur Änderung ihrer Verfassungsschutzgesetze,

- Gesetz zur Änderung der Strafprozessordnung (§ 100i StPO) (BGBl. I 2002, S. 3018),

- Geldwäschebekämpfungsgesetz (BGBl. I 2002, S. 3105),

- Vierundreißigstes Strafrechtsänderungsgesetz ( $\$ 129 \mathrm{~b}$ - Kriminelle und terroristische Vereinigung im Ausland) (BGBI. I 2002, S. 3390),

- Gesetz über die Errichtung des Bundesamtes für Bevölkerungsschutz und Katastrophenhilfe (BGBl. I 2004, S. 630),

- Gesetz zur Neuregelung von Luftsicherheitsaufgaben (BGBl. I 2005, S. 78).

$\mathrm{Zu}$ diesen nationalen Gesetzen traten europäische Normierungen hinzu, die ebenfalls zu berücksichtigen sind aufgrund der inzwischen auch im Bereich der Sicherheitsverwaltung erfolgten Verschränkung von europäischem und nationalem Recht. Zu nennen sind insbesondere:

- Verordnung des Rates über spezifische Maßnahmen zur Terrorismusbekämpfung (ABIEG 2001, Nr. L 344, S. 70),

- Rahmenbeschluss zum Einfrieren von Straftaterträgnissen (ABIEG 2001, Nr. L 182, S. 1),

- Rahmenbeschluss über die Errichtung von Eurojust zur Verstärkung der Bekämpfung der schweren Kriminalität (ABIEG 2002, Nr. L 63, S. 1),

- Rahmenbeschluss zur Terrorismusbekämpfung (ABIEG 2002, Nr. L 164, S. 3),

- Rahmenbeschluss über den Europäischen Haftbefehl und die Übergabeverfahren zwischen den Mitgliedstaaten (ABlEG 2002, Nr. L 190, S. 1).

Die in der vorstehenden Auflistung sichtbar gewordene Normierungswelle hat bislang gewiss noch nicht zu einer völligen Neugestaltung der Sicherheitsarchitektur und des Sicherheitsrechts geführt. Wohl aber sind bereits jetzt schon bemerkenswerte Verschiebungen zu beobachten. 


\section{Es wurden neue Verbots- und Straftatbestände geschaffen.}

Vereine, selbst wenn es sich um Religionsgesellschaften handelt, können verboten werden, nicht nur, wenn ihr Zweck den Strafgesetzen zuwiderläuft oder gegen die verfassungsmäßige Ordnung zielt, sondern auch dann, wenn sie sich gegen den Gedanken der Völkerverständigung richten. Die Gründung einer kriminellen oder terroristischen Vereinigung oder die Beteiligung an ihr steht auch dann unter Strafe, wenn es sich um eine Vereinigung im Ausland handelt.

2. Es wurden europaweit geltende und harmonisierte Bestrafungs-, Verfolgungs- und Kooperationspflichten eingeführt.

Durch europäische Rechtsakte werden die Mitgliedstaaten der Europäischen Union verpflichtet, terroristische Gewalt nach einheitlichen Maßstäben unter Strafandrohung zu stellen. Die Staaten sind ebenso gehalten, daran anknüpfende Folgemaßnahmen zu ergreifen wie grenzüberschreitende Abhöraktionen, sonstige Informationserhebungen, Ermittlungen in Computersystemen oder Inhaftierungen zur Sicherstellung der Auslieferungsfähigkeit.

\section{Die Aufgabenkreise der Polizeien des Bundes, der Nachrichtendienste von Bund und Ländern sowie der Streitkräfte wurden erweitert.}

Die Ermittlungszuständigkeit des Bundeskriminalamtes erstreckt sich nun auch auf Fälle der Computersabotage, sofern diese sich gegen die innere oder äußere Sicherheit der Bundesrepublik Deutschland richtet oder an sicherheitsempfindlichen Stellen auftritt. Die Bundespolizei kann an Bord deutscher Flugzeuge eingesetzt werden und ihre Grenzschutzaufgabe erstreckt sich noch weiter als bisher in den Bereich des Hinterlandes. Den Verfassungsschutzbehörden von Bund und Ländern ist die Aufgabe zugewiesen, auch Informationen über Bestrebungen zu beschaffen, die gegen den Gedanken der Völkerverständigung oder das friedliche Zusammenleben der Völker gerichtet sind. Und die Streitkräfte sind nun mit der luftpolizeilichen Aufgabe betraut, den Eintritt eines besonders schweren Unglücksfalles im Luftraum zu verhindern.

\section{Die Sicherheitsbehörden wurden mit einer Vielzahl neuer Eingriffsbefugnisse aus- gestattet.}

Die Nachrichtendienste sind inzwischen ermächtigt, ihre Datenerhebungsbefugnisse auch zur Beobachtung völkerverständigungswidriger Bestrebungen zu nutzen. Außerdem dürfen sie bei Finanz-, Post-, Telekommunikations- und auch Luftfahrtunternehmen Auskünfte über Konten, Konteninhaber, Gesprächsdaten oder sonstige Umstände des Geschäftsverkehrs einholen. Die Standorte aktiv geschalteter Mobilfunkgeräte sowie die Geräte- und Kartennummern können ermittelt werden. Die Dienste des Bundes dürfen technische Mittel zur Wohnraumüberwachung verwenden, um ihre Mitarbeiter bei Einsätzen zu sichern, die Verfassungsschutzbehörden der Länder darüber hinaus auch in anderen Fällen, so etwa zum Schutz der freiheitlich-demokratischen Grundordnung, der auswärtigen Belange der Bundesrepublik oder auch zur Bekämp- 
fung der organisierten Kriminalität. Diese Maßnahmen dürfen sich nicht nur gegen Verdächtige richten. Auch Drittpersonen können überwacht werden. Es genügt, dass sie in einem bloßen gutgläubigen sozialen Kontakt zu verdächtigen Personen stehen. Das Bundeskriminalamt verfügt mittlerweile über eine eigene Datenerhebungsbefugnis zur Ergänzung vorhandener Sachverhalte sowie zu Zwecken der Auswertung mittels Auskünften oder Anfragen bei öffentlichen und nicht-öffentlichen Stellen. Zur besseren Kontrolle von Ausländern können Identitätsfeststellungen leichter durchgeführt werden. Es existiert nun eine Ermächtigung zur Registrierung biometrischer Merkmale in Ausweisdokumenten, überdies wurde der Katalog von Versagungsgründen für Aufenthaltsgenehmigungen ergänzt und die Rechtsschutzmöglichkeit beschränkt. Zum Zwecke der Rasterfahndung wurde das Sozialgeheimnis gelockert. Zudem wurde der Datenverbund von Nachrichtendiensten, Polizei und Ausländerbehörden weiter ausgebaut. Die Streitkräfte schließlich verfügen nun über eine Befugnis, im Falle eines besonders schweren Unglücksfalles im Luftraum Flugzeuge abzudrängen, zur Landung zu zwingen, den Einsatz von Waffengewalt anzudrohen, Warnschüsse abzugeben und - als letztes Mittel - auch mit Waffengewalt einzuwirken.

\section{Bewertungen und Aufgaben}

\section{Zwischen Apologie und Fundamentalnegation: Bekenntnisse statt Sachkritik}

Die öffentliche Diskussion der nach dem 11. September 2001 geschaffenen Sicherheitsgesetze wird von Extrempositionen geprägt. Auf der einen Seite das apologetische Argument: Der Staat dürfe nicht tatenlos zusehen, wenn das Wüten terroristischer Gewalt die Grundfeste einer freiheitlichen Ordnung wanken lässt. Dabei wird gewiss oft verschwiegen, dass in die Terrorismusbekämpfungsgesetze auch Regelungswerke einflossen, die schon lange in den Schubladen der Ministerialbürokratie lagen, ohne dass auch nur die geringste Chance zur Umsetzung bestanden hätte. Auf der anderen Seite das Verharren im Zustand fundamentaler Verneinung: Durch die Gesetze werde der Rechtstaat attackiert, ja verraten; der Gesetzgeber stelle eine Sicherheit in Aussicht, die nur als totale Sicherheit möglich sei, die Folge sei damit - ein wohl kaum mehr steigerbarer Vorwurf - Staatsterrorismus. Auffällig an dieser Haltung ist der Hang zum Prinzipiellen, zu einer Argumentation, die sich gegenüber den seit der kantischen Frage zu beobachtenden Gewissheitsverlusten im moralischen Denken weitgehend immun zeigt. Eher selten zu finden sind akribisch betriebene Detail- und Tiefenanalysen insbesondere verfassungs- und verwaltungsrechtsdogmatischer Art. Oft verläuft die Suche nach Studien ergebnislos, in denen, auf breiter Faktenbasis beruhend, Notwendigkeiten präzise geprüft, Entscheidungsfolgen plausibel abgeschätzt und Abwägungen sorgfältig vorgenommen werden.

Stattdessen haben die große Formeln Konjunktur, die Zitate aus den klassischen Werken des staatsphilosophischen Denkens, die indessen nur das Problem benennen und beschreiben, es aber nicht lösen und die sich überdies in ihrer Gegenüberstellung neutralisieren: So zutreffend das Humboldtsche Wort ist, ohne Sicherheit sei keine Freiheit, so sehr leuchtet die Hobbessche Erkenntnis ein, dass der, der alle zu schützen 
vermag, auch stark genug ist, alle zu unterdrücken. Gewiss, die Kenntnis der Überlieferung, die Vergegenwärtigung bedeutender Stellungnahmen aus der Geschichte des Staatsdenkens ist unverzichtbar. Dadurch kann der Blick geschärft, können Anregungen gewonnen und Grenzen erkannt werden. Doch jede der klassischen, in den großen Zitaten verdichteten Stellungnahmen ist nicht mehr als eine individuelle Antwort auf Ordnungsprobleme einer bestimmten Zeit, einer spezifischen Epoche. Jede dieser Antworten steht in einem ihr eigenen sozialen, ökonomischen und geistigen Kontext. Sie füllen keinen Fundus vermeintlich überzeitlicher Wahrheiten und Erkenntnisse. Alles andere wäre falsches Historisieren.

\section{Die neuen Aufgaben}

Was aber tut Not, was ist konkret zu tun? Die Balance zwischen Freiheit und Sicherheit - so die häufig zu lesende und hörende Formulierung - ist neu zu justieren. Diese Balance hat eine empfindliche Störung erfahren, dies aber nicht allein durch die neuen, freiheitsbeschränkenden Sicherheitsgesetze, sondern zuerst durch die grauenvollen terroristischen Taten und die dadurch offenkundig gewordene neue Gefährdungslage. Denn Freiheitsverluste entstehen auch dann, wenn sich in signifikanter Weise die Wahrscheinlichkeit erhöht, Opfer nicht-staatlicher Gewalt zu werden. Aber in der Tat: Die aktuelle Aufgabe besteht darin, dass gegenstrebige und spannungsgeladene Verhältnis von individueller Freiheit und sicherheitsgewährender hoheitlicher Macht neu zu bestimmen. Diese Aufgabe erfordert an erster Stelle eine Analyse der Ursachen terroristischer Gewalt. Möglicherweise wurzelt sie in den krassen weltweiten Wohlstandsunterschieden, die immer wieder neue Flüchtlingsströme auslösen. Zudem sollte ein interkultureller Dialog in Gang gesetzt werden, um Wissen zu sammeln, das Vorurteile auflösen und tolerantes Miteinander ermöglichen kann. Aber auch hier ist übertriebenen Erwartungen zu begegnen. Terrorismus, dies zeigt dessen Erforschung, hat oft einen irrationalen Kern, ist selten hauptsächlich bedingt durch Armut und politische Unterdrückung, vielmehr häufig durchsetzt mit ideologischen, religiösen oder sektiererischen Elementen. Und was die gebotene interkulturelle Verständigung angeht, so sind deren Grenzen sehr schnell erreicht, wenn es sich nicht um strategisch denkende Gewalttäter handelt, die eine alternative politische Ordnung durchzusetzen versuchen, sondern aufgrund kultureller Kränkung, Demütigung und Ohnmacht allein danach trachten, nihilistische Zerstörungswut und Rachegefühle auszuleben.

Vorrangig geboten ist dagegen die detailorientierte rechtliche Tiefenanalyse, die keinesfalls allein mit den Parametern der Rechtsstaatlichkeit oder eines vermeintlichen Grundrechts auf Sicherheit zu bewältigen ist. Denn Rechtsstaatlichkeit, material verstanden, ist eine ambivalente, auf Gegenläufigkeiten angelegte Größe. In ihr ist die Pflicht, den Einzelnen vor Schädigungen Dritter zu schützen, ebenso enthalten wie das Gebot, bei Erfüllung dieser Pflicht rechtliche Grenzen zu respektieren. Gleiches gilt für ein angebliches Grundrecht auf Sicherheit, wenn es denn überhaupt mehr sein sollte als eine individualrechtlich verkleidete und pathetisch überhöhte Erinnerung an die staatliche Kernaufgabe des Rechtsgüterschutzes. Denn die Konzeption eines sol- 
chen Rechts lässt die Frage unbeantwortet, mit welchen freiheitsbeschränkenden Mitteln das in ihm angelegte Sicherheitsversprechen einzulösen ist. Vielmehr muss ein Rechtssystem, das auf ein Höchstmaß an Rationalität angelegt ist, sich der Relationen annehmen, die zwischen eingesetzten Mitteln und verfolgten Zielen bestehen. Es muss fragen, ob die gewählten Mittel zielführend sind, ob freiheitsschonendere Wege beschritten werden können, ob die Freiheitsbeschränkungen angesichts der erstrebten Ziele angemessen sind und ob gegebenenfalls unerwünschte Nebenfolgen eintreten. Fehlt es angesichts der Neuheit legislativer Maßnahmen am nötigen Erfahrungswissen, um die Fragen der Geeignetheit, Erforderlichkeit, Angemessenheit und Nebenfolgen begründet oder zumindest plausibel beantworten zu können, so ist nach einem gewissen Zeitraum eine umfassende Evaluierung zwingend geboten. Den parlamentarischen Gremien, die in erster Linie für eine solche Prüfung Verantwortung tragen, sind dabei von den Sicherheitsbehörden alle notwendigen Informationen zur Verfügung zu stellen. Im Terrorismusbekämpfungsgesetz ist eine Evaluierung der neuen nachrichtendienstlichen Eingriffsbefugnisse sogar gesetzlich festgeschrieben. Sie ist, als Ausfluss des verfassungsrechtlich verankerten Verhältnismäßigkeitsgrundsatzes, bei den anderen Eingriffsinstrumenten aber ebenfalls durchzuführen.

Evaluierungen sind indessen auch auf anderen Gebieten vorzunehmen. $\mathrm{Zu}$ überdenken ist das Grundmuster der deutschen Sicherheitsverwaltung, das aus relativ rigiden Trennungsgeboten besteht, aus der prinzipiellen Differenzierung von Polizei und Nachrichtendiensten, Polizei und Militär, Polizei und Privaten, Polizeien des Bundes und der Länder sowie Geheimdiensten des Bundes und Verfassungsschutzämtern der Länder. Es besteht Klärungsbedarf, ob diese überkommenen Trennungen unerlässliche Bestandteile deutscher Freiheitssicherung sind und für alle Zeit so bleiben müssen, so, wie sie in den fünfziger und sechziger Jahren des vergangenen Jahrhunderts mit guten Gründen geschaffen wurden. Oder ob nach einem halben Jahrhundert funktionierender deutscher Rechtsstaatlichkeit diese Trennlinien verschoben oder neu gezogen werden können, ohne dass dabei in unvertretbarer Weise Freiheitsrisiken und -verluste zu befürchten sind. Und ebenfalls zu fragen ist, ob es nur ein krudes Entweder-Oder gibt zwischen einerseits klassischem Polizeirecht, das an die Gefahr-, die Tatverdachtsund die in Ausnahmefällen bestehende Nicht-Störer-Verantwortlichkeit anknüpft, und andererseits einem oftmals diffus und konturenschwach scheinenden, längst mit einer Vielzahl transnationaler Facetten behafteten Präventionsrecht, das Freiheitsbeschränkungen weit im Vorfeld von Gefahr und Verdacht erlaubt und Maßnahmen auch gegen zahlreiche unverdächtige Dritte ermöglicht. Oder ob dieses Vorsorge- und Vorbeugungsrecht nicht doch rechtsstaatlich diszipliniert und freiheitsverträglich ausgestaltet werden kann.

Die gegenwärtig drängenden Themen sind indessen mit der Neustrukturierung sicherheitsrechtlicher Dogmatik, der Überprüfung des sicherheitsbehördlichen Organisationsrechts und der Evaluierung neuer Eingriffsbefugnisse keinesfalls abschließend benannt. Von besonderer Brisanz ist vielmehr die Frage, ob bei der Erfüllung der staatlichen Pflicht zum Rechtsgüterschutz nicht eine absolute, mithin unverschiebbare und unüberwindbare Grenze zu beachten ist, die verfassungsrechtlich durch die Menschenwürdegarantie des Grundgesetzes statuiert wird. Die im letzten Jahr ergangene 
Entscheidung des Bundesverfassungsgerichts zur akustischen Wohnraumüberwachung, dem so genannten »Großen Lauschangriff«, kann so gedeutet werden: In ihr wurde - zumindest für den Bereich heimlicher Überwachungsmaßnahmen des Staates - herausgestellt, dass der Schutz der Menschenwürde einen unantastbaren Kernbereich persönlicher Lebensgestaltung sichert, in den einzugreifen den Sicherheitsbehörden selbst dann versagt ist, wenn noch so starke und gewichtige Allgemeininteressen wie etwa die Effektivität der Strafrechtspflege nach einem solchen Eingriff verlangen.

Verliert aber - und dabei handelt es sich um eine unausweichliche Folgefrage - die durch die Menschenwürdegarantie des Grundgesetzes benennbare Grenze sicherheitsbehördlichen Handelns dann ihre Absolutheit, wenn ihre Beachtung selbst eine Würdeverletzung zur Folge hat, wenn also der Schutz der Würde des einen unweigerlich mit der Verletzung der Würde eines anderen einhergeht? Um dies zu konkretisieren: Darf etwa würdeverletzender Zwang zur Informationsgewinnung gegen einen Entführer eingesetzt werden, wenn eine solche würdewidrige »Präventivfolter « gegen den Täter als einziges Mittel erscheint, um die Würde des Entführungsopfers zu schützen? Oder: Ist als letztes Mittel die Tötung unschuldiger Flugzeugpassagiere erlaubt, wenn diese Passagiere im Falle eines als Waffe benutzten Flugzeuges mit an Sicherheit grenzender Wahrscheinlichkeit auch dann zu Tode kämen, wenn der Staat nicht eingriffe, demgegenüber bei und infolge eines staatlichen Abschusses des Flugzeuges zumindest die Chance bestünde, eine Vielzahl von Menschen zu retten, die ohne staatliches Handeln indessen an dem von den Flugzeugentführern gewählten Absturzort zu Tode kämen? Sich diesen Fragen zuzuwenden, entspringt keinesfalls, wie dies gelegentlich zu lesen ist, der Lust am aufregenden und schweren Denken oder der mangelnden Resistenz gegenüber einer vermeintlichen Faszination des Ausnahmezustandes. Es sind konkrete Anlässe und Erfahrungen, die diese Fragen in den Vordergrund gerückt haben. Allerdings führen diese Fragen in unwegsame und schluchtendurchzogene Grenzregionen des Rechts, die nicht in der Erwartung betreten werden sollten, dort bündige, klare und restlos befriedigende Antworten zu finden. Gleichwohl sollte nicht die Hoffnung aufgegeben werden, dass auch dort das zunächst unmöglich Erscheinende am Ende dennoch möglich ist, nämlich mit rechtstaatlicher Vernunft das spannungsträchtige Gegenüber von hoheitlichem Sicherheitsversprechen und individuellem Freiheitsverlangen auch in diesen bedrückenden Grenzbereichen in einer halbwegs erträglichen Balance zu halten.

\section{Literaturhinweise}

Achelpöhler, W./Niehaus, H., Rasterfahndung als Mittel zur Verhinderung von Anschlägen islamistischer Terroristen in Deutschland, DÖV 2003, S. 49 ff.

Albers, M., Terrorismusbekämpfungsgesetz und Datenschutz, in: Schubert/Reusch/Jesse (Hrsg.), Informatik bewegt. Informatik 2002 - 32.Jahrestagung der Gesellschaft für Informatik e.V., Ergänzungsband 2002.

Albrecht, P.-A., Die vergessene Freiheit. Strafrechtsprinzipien in der europäischen Sicherheitsdebatte, 2003. 
Baldus, M.,

- Nachrichtendienste - Beobachtung völkerverständigungswidriger Bestrebungen, ZRP 2002, S. 400 ff.

- Der Beitrag des Europäischen Polizeiamtes (Europol) zur Bekämpfung des Terrorismus, in: Koch (Hrsg.), Terrorismus - Rechtsfragen der äußeren und inneren Sicherheit, 2002, S. $121 \mathrm{ff}$.

- Präventive Wohnraumüberwachung durch Verfassungsschutzbehörden der Länder. Ein gesetzestechnisch unausgegorenes und verfassungsrechtlich zweifelhaftes Mittel zur Terrorismusbekämpfung, NVwZ 2003, S. 1289 ff.

- Streitkräfteeinsatz zur Gefahrenabwehr im Luftraum, NVwZ 2004, S. 1278 ff.

- Überwachungsrecht unter Novellierungsdruck, in: Schaar (Hrsg.), Folgerungen aus dem Urteil des Bundesverfassungsgericht zur akustischen Wohnraumüberwachung: Staatliche Eingriffe auf dem Prüfstand?, 2004, S. 9 ff.

Bartelt, S./Zeitler, H.E., »Intelligente Sanktionen« zur Terrorismusbekämpfung in der EU, EuZW 2003, S. $712 \mathrm{ff}$.

Bizer, J., Politik der inneren Sicherheit, DuD 2002, S. $741 \mathrm{ff}$.

Brugger, W.,

- Vom unbedingten Verbot der Folter zum bedingten Recht auf Folter?, JZ 2000, S. 165 ff.

- Gewährleistung von Freiheit und Sicherheit im Lichte unterschiedlicher Staats- und Verfassungsverständnisse, in: VVDStRL 2004, S. $101 \mathrm{ff}$.

Bubnoff, E.v., Terrorismusbekämpfung - eine weltweite Herausforderung, NJW 2002, 2672 ff.

Bull, H-P.,

- Freiheit und Sicherheit angesichts terroristischer Bedrohung. Bemerkungen zur rechtspolitischen Diskussion, in: Möllers/van Ooyen (Hrsg.), Jahrbuch Öffentliche Sicherheit, 2002/03, S. 265 ff.

- Polizeiliche und nachrichtendienstliche Befugnisse zur Verdachtsgewinnung, in: Osterloh/Schmidt/ Weber (Hrsg.), FS für Peter Selmer, 2004, S. 29 ff.

Calliess, C., Gewährleistung von Freiheit und Sicherheit im Lichte unterschiedlicher Staats- und Verfassungsverständnisse, DVB1 2003, S. 1096 ff.

Denninger, E.,

- Freiheit durch Sicherheit. Anmerkungen zum Terrorismusbekämpfungsgesetz. Strafverteidiger 2002, S. $96 \mathrm{ff}$.

- Freiheit durch Sicherheit? Anmerkungen zum Terrorismusbekämpfungsgesetz, Das Parlament, 2002, Heft 11, 8./15. März, S. 1 ff.

- Fünf Thesen zur »Sicherheitsarchitektur« insbesondere nach dem 11. September 2001, in: Möllers/ van Ooyen (Hrsg.), Jahrbuch Öffentliche Sicherheit, 2002, S. 253 ff.

- Freiheit durch Sicherheit?, in: Koch, H.-J. (Hrsg.), Terrorismus - Rechtsfragen der äußeren und inneren Sicherheit, 2002, S. 83 ff.

- Verfassungsrechtliche Grenzen des Lauschens, ZRP 2004, S. 101 ff.

Düx, H., Globale Sicherheitsgesetze und weltweite Erosion von Grundrechten, ZRP 2003, S. $189 \mathrm{ff}$.

Erbel, G., Die öffentliche Sicherheit im Schatten des Terrorismus, Das Parlament, 2002, 11, 8./ 15. März 2002.

Fischer, M.G., Terrorismusbekämpfung durch die Bundeswehr im Inneren Deutschlands, JZ 2004, S. $376 \mathrm{ff}$.

Glaeßner, G.-J., Sicherheit in Freiheit. Die Schutzfunktion des demokratischen Staates und die Freiheit seiner Bürger, 2003

Gusy, C.,

- Gemeindienstliche Aufklärung und Verfassungschutz, in: Koch, H.-J. (Hrsg.), Terrorismus - Rechtsfragen der äußeren und inneren Sicherheit, 2002, S. 93 ff.

- Die Vernetzung innerer und äußerer Sicherheitsinstitutionen in der Bundesrepublik Deutschland, in: Weidenfeld, W. (Hrsg.), Herausforderung Terrorismus - Die Zukunft der Sicherheit, 2004, S. 197 ff.

- Gewährleistung von Freiheit und Sicherheit im Lichte unterschiedlicher Staats- und Verfassungsverständnisse, in: VVDStRL 2004, S. $151 \mathrm{ff}$. 
- Auswirkungen des Lauschangriffsurteils außerhalb der strafprozessualen Wohungsüberwachung, in: Schaar (Hrsg.), Folgerungen aus dem Urteil des Bundesverfassungsgericht zur akustischen Wohnraumüberwachung: Staatliche Eingriffe auf dem Prüfstand?, 2004, S. 9 ff.

Hassemer, W., Zum Spannungsverhältnis von Freiheit und Sicherheit. Drei Thesen, in: Vorgänge. Zeitschrift für Bürgerrechte und Gesellschaftspolitik, Nr. 159, September 2002

Hetzer, W., Terrorismus und Gesetzgebung. Polizei und Nachrichtendienste: Kooperation oder Konfrontation? ThürVBl, 2002, S. $251 \mathrm{ff}$.

Hirschmann, K./Leggemann, C. (Hrsg.), Der Kampf gegen den Terrorismus. Strategien und Handlungserfordernisse in Deutschland, 2003

Hochhuth, M., Militärische Bundesintervention bei inländischem Terrorakt. Verfassungsänderungspläne aus Anlass der Flugzeugentführungen vom 11. September 2001, NZWehrR 2002, 154 ff.

Höche, T., Neue gesetzliche Regelungen zur Bekämpfung des Terrorismus und der Geldwäsche, Die Bank, 2002, S. $196 \mathrm{ff}$.

Hoffmann-Riem, W., Freiheit und Sicherheit im Angesicht terroristischer Anschläge. Zeitschrift für Rechtspolitik, 2002, S. 497 ff.

Horn, H.-D., Vorbeugende Rasterfahndung und informationelle Selbstbestimmung, DÖV 2003, S. $746 \mathrm{ff}$.

Humanistische Union (Hrsg.), Innere Sicherheit als Gefahr, 2003

Jahn, J., Verschärfte Finanzkontrollen nach Terroranschlägen, ZRP 2002, S. 109 ff.

Kersten, J., Die Tötung von Unbeteiligten, NVwZ 2005, S. $661 \mathrm{ff}$.

Koch, H.-J. (Hrsg.), Terrorismus - Rechtsfragen der äußeren und inneren Sicherheit, 2002

- ders./John, Atomrechtliche Fragen der Sicherheit und Sicherung von Kernkraftwerken nach den Terroranschlägen vom 11. September 2001 in den USA, DVB1 2002, S. 1578 ff.

Krings, G./Burkiczak, C., Bedingt abwehrbereit - Verfassungs- und völkerrechtliche Aspekte des Einsatzes der Bundeswehr zur Bekämpfung neuer terroristischer Gefahren im In- und Ausland, DÖV 2002, S. $501 \mathrm{ff}$.

Lacqueur, W., Krieg dem Westen. Terrorismus im 21. Jahrhundert, 2003

Limbach, J., Ist die kollektive Sicherheit der Feind der individuellen Freiheit?, 2002

Lutze, C., Abwehr terroristischer Angriffe als Verteidigungsaufgabe der Bundeswehr, NZWehrR 2003, S. $101 \mathrm{ff}$.

Marx, R., Folter: eine zulässige polizeiliche Präventionsmaßnahme?, KritV 2004, S. 278 ff.

Mehde, V., Terrorismusbekämpfung durch Organisationsrecht, JZ 2005, S. 815 ff.

Münkler, H., Die neuen Kriege, 2002.

Nehm, K., Ein Jahr danach. Gedanken zum 11. September 2001, NJW 2002, S. 2665 ff.

Nolte, M., Die Anti-Terror-Pakete im Lichte des Verfassungsrechts, DVB1 2002, S. $573 \mathrm{ff}$.

Ossenbühl, F., Terroristische Angriffe auf Kernkraftwerke - aus rechtlicher Sicht, NVwZ 2002, S. $290 \mathrm{ff}$.

Paeffgen, H-U., »Vernachrichtendienstlichung« von Strafprozeß- (und Polizei-)recht im Jahr 2001, Strafverteidiger 2002, S. $336 \mathrm{ff}$.

Petri, T. B., Im Schatten des Leviathan - Zum Verhältnis von Sicherheit und Freiheit anhand von Beispielen aus der TK-Überwachung. RDV 2003, S. 16 ff.

Pieroth/Hartmann, Der Abschuss eines Zivilflugzeugs auf Anordnung des Bundesministers für Verteidigung, Jura 2005, S. 729 ff.

Rau, M., Country Report on Germany, in: Walter/Vöneky/Röben/Schorkopf (Hrsg.), Terrorism as a Challenge for National and International Law: Secuity versus Liberty, 2004, S. $311 \mathrm{f}$.

Rublack, S., Schwerpunkt. Terrorismusbekämpfungsgesetz: Neue Befugnisse für die Sicherheitsbehörden, DuD 2002, S. $202 \mathrm{ff}$.

Schmidt-Jortzig, E., Verfassungsänderung für Bundeswehreinsätze im Innern Deutschlands?, DÖV 2002, S. $773 \mathrm{ff}$.

Sendler, H., Nochmals: Terroristische Angriffe auf Kernkraftwerke, NVwZ, 2002, S. 681 ff. 
Strossen, N., Der Schutz von nationaler Sicherheit und individueller Freiheit - eine amerikanische Perspektive nach dem 11. September, in: Humanistische Union (Hrsg.), Innere Sicherheit als Gefahr, 2002, S. $65 \mathrm{ff}$.

Vennemann, N., Contry Report on the European Union, in: Walter/Vöneky/Röben/Schorkopf (Hrsg.), Terrorism as a Challenge for National and International Law: Secuity versus Liberty, 2004, S. $218 \mathrm{ff}$. Weidenfeld,W., Herausforderung Terrorismus - Die Zukunft der Sicherheit, 2004

Werthebach, E., Deutsche Sicherheitsstrukturen im 21. Jahrhundert, in: APuZ 2004, B44, S. 5 ff.

Wiefelspütz, D., Sicherheit vor den Gefahren des internationalen Terrorismus durch den Einsatz der Streitkräfte?, NZWehrR 2003, S. 45 ff.

Wilkesmann, P., Terroristische Angriffe auf die Sicherheit des Luftverkehrs, NVwZ 2002, S. 1316 ff. Wittreck, F., Menschenwürde und Folterverbot, DÖV 2003, S. 873 ff.

Wolff, H.A., Der verfassungsrechtliche Rahmen für den Einsatz der Bundeswehr im Innern zur Terrorismusbekämpfung und zum Schutz ziviler Objekte, ThürVB1 2003, S. 176 ff.

Ziercke, J., Neue Sicherheitsarchitektur für Deutschland, Kriminalistik 2002, S. 346 ff. 